\title{
Attention and the processing of emotional words: Dissociating effects of arousal
}

\author{
Jennifer M. Aquino and Karen M. Arnell \\ Brock University, St. Catharines, Ontario, Canada
}

\begin{abstract}
There is debate as to whether emotionally charged words receive preferential attentional processing in normal individuals. Using a digit-parity task, Harris and Pashler (2004) found that threat-related words captured attention on only the first trial, suggesting no attention capture for emotional words aside from an initial surprise reaction. We examined whether sexually explicit words would be more effective at capturing attention in a similar task. Our results with threat words replicated those of Harris and Pashler in that threat words did not lead to an increase in reaction time (RT) on the parity task, relative to emotionally neutral words. However, sexual words led to a marked increase in RTs for over 100 trials. Words' arousal ratings, but not their valence ratings, predicted the amount of interference. Parity RTs for individual words were also related to memory for the word on a surprise memory test. Sexually explicit words may have more potential to capture attention than do threat-related words. The words presented here as distractors in the digit-parity task, along with their corresponding valence ratings, arousal ratings, and digit-parity RTs, can be downloaded from www.psychonomic.org/archive.
\end{abstract}

In the marketing world, there is a common assumption that sex sells, due to the ability of sexual material to capture viewers' attention. Although various studies have explored the attentional capture of "emotionally charged stimuli" such as fear/threat stimuli, or stimuli consistent with our fears or goals (e.g., Dijksterhuis \& Aarts, 2003; Harris \& Pashler, 2004; Williams, Mathews, \& MacLeod, 1996), little research has examined the effect of sexually explicit stimuli on attention, despite the fact that sexually explicit stimuli can also be classified as emotionally charged.

One paradigm used to examine possible attention capture by emotional stimuli is the digit-parity paradigm. Wolford and Morrison (1980) showed participants a display consisting of two digits flanking a centrally presented word. The participants were asked to make a speeded judgment about whether the parity of the two digits matched (both odd or both even) or did not match (one odd and the other even), while ignoring the irrelevant center word. When the participant's last name was presented between the digits, responses to the digits were slowed significantly, suggesting that the presentation of the participant's last name as a distractor attracted his or her attention.

More recently, using the same digit-parity task, Harris and Pashler (2004) showed that when negatively emotionally charged words (fear, threat, and anxiety words) were presented between the digits, only the first presentation of the emotionally charged word elicited an increase in reaction time (RT). When an equal number of neutral and emotionally charged words were presented randomly within a block, Harris and Pashler found that the mean RTs did not differ reliably. Therefore, Harris and Pashler concluded that their results support the need to focus on the initial appearance of emotionally charged stimuli to detect any type of attentional capture, and suggested that emotional stimuli are not so special after all, in terms of attracting attention. However, it is possible that the use of negatively charged words as potential distractors did not result in a strong enough manipulation of emotional salience, given the use of a nonclinical sample. Although there is good evidence suggesting that anxiety patients do show preferential attentional capture for anxiety-related words (Williams et al., 1996), there is accumulating evidence suggesting that fear/threat words are relatively weak emotional stimuli, relative to taboo stimuli, when shown to unselected undergraduate participants. For example, Arnell, Killman, and Fijavz (in press) showed that taboo words were able to capture participants' attention and reduce accuracy on subsequent targets when presented as distractors in rapid serial visual presentation, but that anxiety and threat words were not. Similarly, longer color-naming times for taboo versus neutral words have been demonstrated in emotional Stroop tasks (MacKay et al., 2004). However, elevated color-naming RTs to fear/danger words are often not observed in emotional Stroop tasks with these same subject populations (Williams et al., 1996). Larsen, Mercer, and Balota (2006) have also recently observed that many past studies of emotional Stroop tasks have confounded lexical characteristics of words such as frequency and length with emotional valence. Therefore, word choice alone may be responsible for the fleeting effect observed by Harris and Pashler. The use of more shocking words as distractors

K. M.Arnell, karnell@brocku.ca 
in a digit-parity task may be more effective at capturing participants' attention.

\section{The Present Investigation}

In our digit-parity task, the threat and neutral distractor words were selected from the same set of words used by Harris and Pashler (2004). Most of the sexually explicit words were selected from the word set used by Anderson (2005). Attentional capture by emotional stimuli may not be the result of the emotionality of the stimuli per se, but of the fact that the emotional stimuli form a tighter category than do random neutral words (McKenna \& Sharma, 1995). To address this issue, as well as the literature suggesting that participants may be distracted by concernrelevant stimuli (Dalgleish, 1995; Williams et al., 1996), we added a control category of school words, which not only form a single category but are also concern relevant to our sample of undergraduate students. All four word types (neutral, threat, school, and sexual) will be presented randomly within a block of trials. Memory for the distractor words presented during the parity task, as well as valence and arousal ratings for the distractor words, will also be examined, in an attempt to elucidate the nature of the interference.

\section{METHOD}

\section{Participants}

Thirteen Brock University male $(n=7)$ and female $(n=6)$ undergraduate students ranging in age from 18 to 22 years $(M=$ 19.7 years) participated for either payment or course credit.

\section{Design}

The experiment consisted of two blocks of 100 parity trials each, with 20 practice trials prior to Block 1 . The practice block used neutral words that were different from the neutral words presented in Blocks 1 and 2. In Block 1, 25 threat-related and 25 neutral words randomly selected from McKenna and Sharma $(1995)^{1}$, as well as 25 sexual words and 25 school words, were each presented once. (The words used here, along with the mean valence rating, mean arousal rating, and mean parity RT for each word, are available at the Psychonomic archive, www.psychonomic.org/archive.) The words were chosen randomly by the computer, with the constraint that each word category would be presented five times every 20 trials. In Block 2, the same 100 words were again shown in random order, with the same constraint.

\section{Apparatus and Stimuli}

The experiment was controlled using E-Prime software (Schneider, Eschman, \& Zuccolotto, 2002) running on a Sony VIAO desktop computer with a 17-in. color monitor. Displays consisted of two digits flanking a word, and were viewed from an unfixed distance of approximately $55 \mathrm{~cm}$

The stimuli were black against a light gray background. The digits and words were presented in 16-point bold Courier New font, and all of the words were capitalized. The words were four to eight letters long and subtended approximately $1^{\circ}$ in height and $2^{\circ}$ to $5^{\circ}$ in width. The digits were spaced $10.5 \mathrm{~cm}$ apart $\left(11.5^{\circ}\right)$ on all trials. Only the digits $2,3,5$, and 8 were used as stimuli. The digits were randomly paired, with the constraint that on half of the trials, the pair of digits had the same parity.

\section{Procedure}

Each parity trial began with the presentation of a black fixation cross in the center of the computer screen for $500 \mathrm{msec}$, followed by a 500-msec blank interval before the presentation of a word with a digit to the left and right of it. The words and digits remained on the screen until a response was made. The participants were told to press the $\mathrm{M}$ key if the digits matched, and to press the $\mathrm{N}$ key if the digits did not match. The participants were asked to respond as quickly as possible, while being accurate, and to ignore the center words. No indication was given about the impending recognition test.

Immediately following the parity task, the participants were given a piece of paper containing an alphabetic arrangement of the 100 words presented during the parity task, plus 8 foil words for each word category that had not been presented. The participants were told that some of the words on the list were presented as distractors in the parity task and that they should check off as many or as few words as they remembered seeing, going through the list in any order, with no time constraints.

Next, the participants rated the valence and arousal of each of the 100 words from the parity task, which were presented in random order. Using a 7-point Likert scale for each rating, the participants gave each word a valence rating (negative to positive) and an arousal rating (low to high). The participants were specifically instructed that valence represented the degree to which they considered a word to be negative to neutral to positive, with 1 being most negative, 4 being neutral, and 7 being most positive. They were further instructed that arousal did not refer to sexual arousal per se, but to the shock value of the word (e.g., the participants were told that being chased by a dog, or winning the lottery, could represent a situation that was highly arousing).

\section{RESULTS}

\section{Digit-Parity Reaction Time}

Figure 1 shows the mean RT (in milliseconds) for the parity task for each word category as a function of block. A $4 \times 2$ ANOVA was performed on mean RT scores, with word category (school, sexual, threat, and neutral), and block (1 or 2) as within-participants variables. The analysis revealed a significant main effect of word category $[F(3,36)=9.11, p<.01]$, with significantly longer RTs for sexual words relative to all other words (all $p \mathrm{~s}<.05$ ), but no other significant differences between word categories (all $p \mathrm{~s}>.05$ ). The ANOVA produced no main effect of block $[F(1,12)=2.88, p>.12]$, but did show a significant block $\times$ word category interaction $[F(3,36)=4.12$, $p<.05$ ], given that the difference in RT between sexual words and all other word categories was larger in Block 1 than in Block 2. A one-way ANOVA on Block 1 RTs revealed a significant effect of word category $[F(3,36)=$ $8.96, p<.01]$. Tukey HSD tests showed significant differences between sexual words and all other word categories (all $p \mathrm{~s}<.05$ ), with no difference in RTs for threat, neutral, and school words (all $p \mathrm{~s}>.05$ ). For Block 2, an ANOVA also revealed a significant effect of word category $[F(3,36)=3.43, p<.05]$. Tukey HSD tests showed a significant difference between sexual and threat words only $(p<.05)$, and a marginally significant difference between sexual and school words $(p<.06)$.

A $4 \times 2$ item analysis was performed, with word category as the between-participants variable and block as the within-participants variable. The analysis also revealed a significant main effect of block $[F(1,96)=23.43, p<$ $.01]$ and word category $[F(3,96)=12.14, p<.01]$, but no significant interaction $[F(3,96)=2.28, p>.05]$. Tukey HSD tests revealed significantly longer RTs for sexual words relative to all other words (all $p \mathrm{~s}<.01$ ), with no other significant differences (all $p$ s $>.95$ ). 


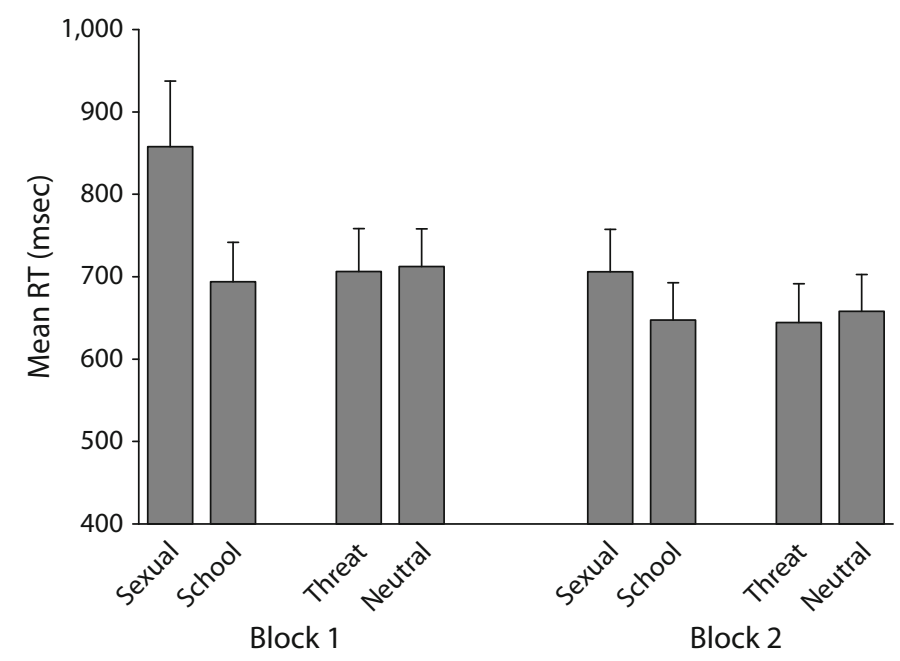

Figure 1. Mean digit-parity reaction time for each word category as a function of block. Error bars represent the standard error for each mean.

To further examine the difference in RT between the emotional and neutral words within a block, we divided each block into five miniblocks containing 20 trials each ( 5 trials for each of the four word types). Figure 2A shows Block 1 mean RT difference scores (emotional-word RTs neutral-word RTs) for the word set created for this experiment (sexual - school) and those taken from Harris and Pashler (2004) (threat - neutral). The pattern in Figure 2A suggests that the RT slowing for sexual words relative to school words lasted throughout the block of 100 trials, and was not simply present for the first few presentations of sexual words. A $2 \times 5$ repeated measures ANOVA confirmed that sexual words were associated with longer RTs than were school words, overall $[F(1,12)=13.64, p<.01]$. There was also a main effect of miniblock $[F(4,48)=4.22$, $p<.01]$, where RTs decreased across the block. Importantly, the emotion $\times$ miniblock interaction did not yield significant results $(F<1)$, suggesting that the difference in RTs between the sexual and school words remained the same across blocks. An ANOVA confirmed that there was no difference in RTs for threat-related and neutral words, overall $(F<1)$. Furthermore, the failure to observe an RT difference remained across miniblocks $(F<1)$. Figure 2B shows the same RT difference scores for Block 2 (Trials 100 to 200). Block 2 RTs again revealed longer RTs for sexual words than for school words $[F(1,12)=6.44, p<.05]$, and no emotion $\times$ miniblock interaction $(F<1)$. Block 2 RTs again showed no difference in RTs for threat-related and neutral words overall $(F<1)$, and this remained constant across the miniblocks $(F<1)$.

Finally, to test whether Harris and Pashler's (2004) effect of finding an increase in RT for the first presentation of a threat word was replicated, we compared the initial RT for each word category using a one-way ANOVA. No significant difference in first-trial RTs was observed $[F(3,36)=2.07, p>.12]$ across the word categories. Furthermore, no significant difference in neutral and threat first-trial RTs was observed when these were compared directly $[t(12)=1.86, p>.05]$.

\section{Accuracy}

Errors occurred on $7.1 \%$ of all trials, and were slightly higher for the sexual (7.5\%) and school (8.5\%) words than for the threat $(6 \%)$ and neutral $(6.5 \%)$ words. A $4 \times 2$ repeated measures ANOVA performed on the error rates revealed no main effect of word category $[F(3,36)=2.06$, $p>.12]$ or of block $[F(1,12)=4.15, p>.05]$ and no interaction of word category and block $(F<1)$.

\section{Frequency and Word Length}

Using Kučera and Francis (1967), we could not obtain word frequency estimates for 10 of the sexual words. Across word categories, word frequency estimates for the other 90 words ranged from 1 to 492 per million, with a mean frequency of 58.97 per million. The frequency of a word did not predict parity RT on trials in which that word was presented $[r(89)=-.09, p>.42]$. Furthermore, a comparison of the mean parity RTs for the 10 most frequent words $(714 \mathrm{msec})$ and the 10 least frequent words (722 msec) produced no significant difference $(t<1)$. Across word types, word length ranged from four to eight letters $(M=5.5)$. When word length was correlated with parity RT on trials containing that word, no significant relationship was observed $[r(98)=-.04, p>$.69]. Furthermore, the mean parity RT for the longest words (seven or eight letters) did not differ significantly from the mean RT for the shortest words (four letters) $(t<1)$.

\section{Word Ratings}

Mean arousal and valence ratings were calculated for each word, averaging across participants. A one-way ANOVA showed that mean arousal ratings differed across word categories $[F(3,96)=195.46, p<.01]$. Tukey HSD tests showed that sexual words $(M=5.18)$ were rated as 

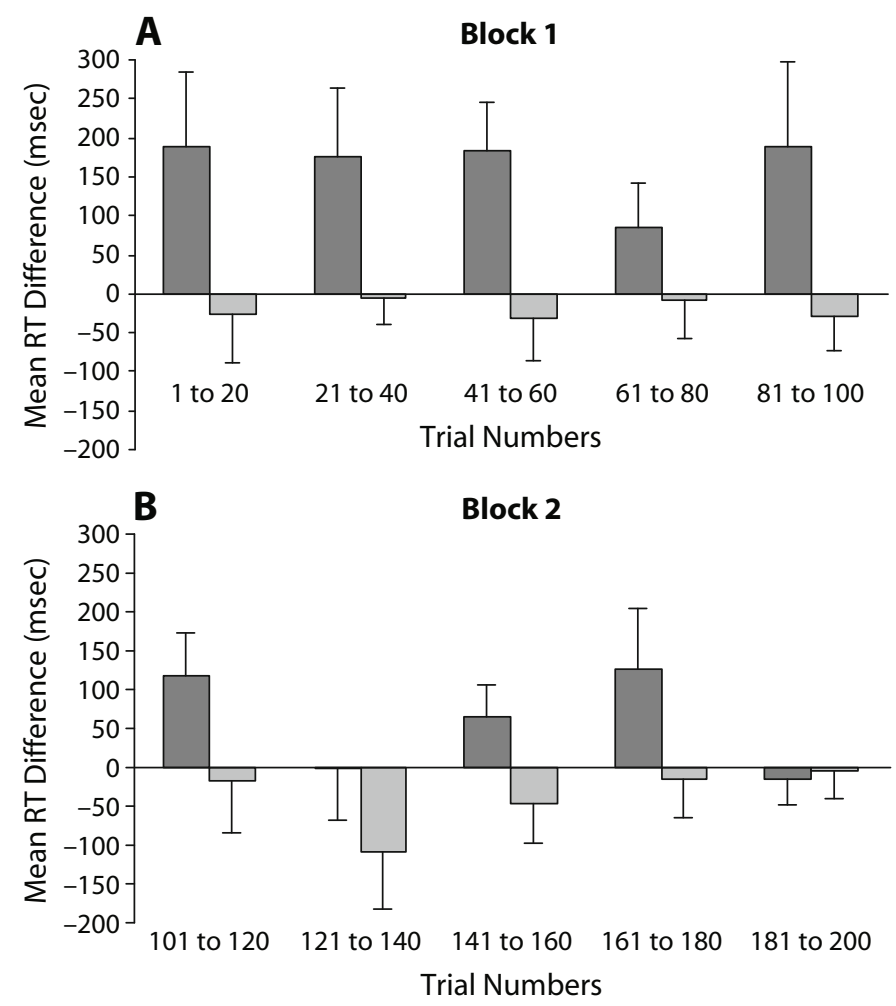

Figure 2. Panel A shows Block 1 mean RT difference scores, and panel B shows Block 2 mean RT difference scores. Dark bars represent parity RTs on sexual-word trials - parity RTs on school trials. Light bars represent parity RTs on threat-word trials - parity RTs on neutral trials. Error bars represent the standard error for each mean.

significantly more arousing than school words $(M=2.34)$, threat-related words $(M=4.44)$, and neutral words $(M=$ 2.31) (all $p \mathrm{~s}<.05)$. Threat-related words were rated as significantly more arousing than school and neutral words ( $p$ s $<.05)$, but school and neutral words did not differ $(p>$ .99). A one-way ANOVA showed that mean valence ratings also differed across word categories $[F(3,96)=50.29, p<$ $.01]$. Tukey HSD tests showed that threat-related words $(M=2.04)$ were rated as significantly more negative than sexual words $(M=4.32)$, school words $(M=4.14)$, and neutral words $(M=4.37)$ (all $p$ s $<.05)$. No other comparisons were significant (all $p \mathrm{~s}>.73$ ).

Arousal and valence ratings for each word were correlated with the parity RT on trials in which that word was presented. Arousal ratings significantly predicted parity RTs $[r(98)=.38, p<.001]$, where higher word arousal ratings were associated with longer parity RTs. This was true even when the variability due to valence ratings was partialed out (semipartial $r=.44, p<.001$ ). However, valence ratings did not predict parity RTs $[r(98)=.15$, $p>.13]$, despite the fact that arousal and valence ratings were negatively correlated $[r(98)=-.31, p<.001]$. It is also possible that very negative and very positive words may have the largest effect on parity RTs. To test this possibility, we created valence extremity scores using the absolute difference between valence ratings and the midpoint on the rating scale (4). Valence extremity scores also did not predict parity RTs $[r(98)=-.09, p>.19]$, despite the fact that arousal and valence extremity ratings were negatively correlated $[r(98)=-.60, p<.001]$. Arousal ratings significantly predicted parity RT over and above valence extremity scores (semipartial $r=.54, p<.001$ ).

\section{Memory Checklist}

The number of times each word was checked as being remembered on the checklist was summed across the participants (maximum score 13/13 participants). Table 1 shows the mean number of checks received by a word on the memory checklist as a function of word category,

Table 1

Mean Number of Checks Received by a Word on the Memory Checklist As a Function of Word Category and Whether the Word Appeared As a Distractor During the Digit-Parity Task (Hit) or Was Not Presented During the Digit-Parity Task (False Alarm)

\begin{tabular}{crcc}
\multicolumn{4}{c}{ (False Alarm) } \\
\hline Word Category & Hits & False Alarms & Memory \\
\hline Sexual & 10.76 & 2.63 & 8.13 \\
School & 3.88 & 1.38 & 2.50 \\
Threat & 6.20 & 1.38 & 4.82 \\
Neutral & 3.52 & 0.63 & 2.89 \\
Overall & 6.09 & 1.50 & 4.59
\end{tabular}

Note-Memory is defined as the difference between hits and false alarms. 
and whether the word appeared as a distractor during the digit-parity task (hit) or was not presented during the digit-parity task (false alarm). We calculated memory performance by subtracting the mean number of false alarms from the number of hits for each word category. A one-way ANOVA confirmed that memory performance differed significantly across word categories $[F(3,96)=$ $39.35, p<.001]$. Tukey HSD tests showed better memory for sexual words relative to all other word categories (all $p$ s $<.001)$. School and neutral words were recognized equally as often $(p>90)$, but both were recognized less often than threat-related words.

Arousal ratings for a word, but not its valence ratings, predicted the memory for each word $[r(98)=.73, p<$ .001 , and $r(98)=-.08, p>.45$, respectively]. When memory for each of the words was correlated with parity RTs on trials in which that word was presented, a significant relationship was observed $[r(98)=.36, p<.001]$. Words that were remembered more often were associated with longer parity RTs, suggesting that on trials in which distractor words are encoded into memory, parity performance suffers. Indeed, arousal ratings no longer predicted RTs once memory for the words was partialed out [semipartial $r=.17 ; t(97)=1.78, p>.08$ ] . This pattern suggests a direct relationship between encoding of the word and parity RTs and an indirect relationship between arousal rating and RTs that is mediated by encoding of the distractor word (see Figure 3).

\section{DISCUSSION}

Only the sexual words, relative to all other word categories (threat, neutral, and school), led to an increase in parity RTs, and sexual words were encoded into memory for later recall more so than any other word category. Furthermore, arousal but not valence ratings of the words predicted this encoding and the parity RTs.

Harris and Pashler (2004) showed that the first presentation of a threat-related word elicited a marked increase in RT for the parity task, but that this effect habituated quite rapidly, becoming virtually undetectable by the second presentation. They attributed the performance disruption on the first trial to a momentary surprise response, and suggested that previous findings of attentional capture to

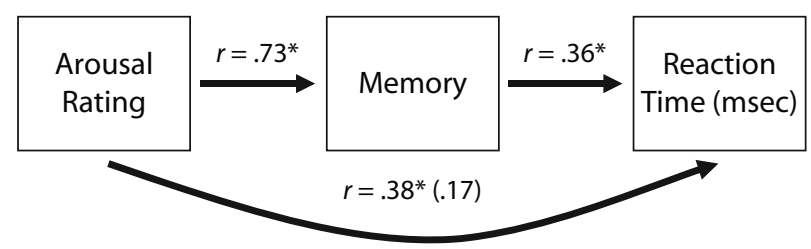

Figure 3. Model showing the interrelationship of digit-parity RT, arousal ratings, and memory performance. Significant relationships are denoted by an asterisk. The value in brackets represents the unique relationship between arousal ratings and parity RTs once memory has been accounted for (semipartial $r$ ). The model suggests that the relationship between arousal ratings and parity RT is mediated by conscious encoding of the word. emotionally charged stimuli (e.g., Wolford \& Morrison, 1980) may simply have resulted from averaging a long RT on the first or second presentation of the word into the overall mean. When the threat-related words were presented randomly on half of the trials with neutrals words, Harris and Pashler found that mean parity RT did not differ for the two conditions. The results of the present study replicate Harris and Pashler's, in that when threat-related words were presented randomly within a block, no increase in parity RT was observed, relative to emotionally neutral words. However, a marked increase in parity RT was observed when sexual words were presented. Furthermore, in contrast to the results of Harris and Pashler, this effect can be seen well beyond the first presentation of a sexual word, with increased RTs for the sexual words lasting well over 100 trials. Even when participants were shown the set of sexual words in Block 2 that were previously presented in Block 1, an increase in RT for the sexual words was still noted (although smaller than for Block 1), suggesting that the results cannot be explained simply by a momentary surprise response.

In the present investigation, in contrast to the results of Harris and Pashler (2004), the first presentation of a threat word did not lead to an increase in parity RT relative to an emotionally neutral word. The lack of significance is perhaps not surprising, given that individual trial RTs are very noisy, needing an unusually large $N$ to be able to show reliable differences. However, in the present study, first threat-trial RTs were numerically smaller than first neutral-trial RTs, suggesting that the difference was not simply a power issue. In Block 1 of Harris and Pashler's study, the emotional stimuli were presented very infrequently ( 2 trials out of 50); thus, perhaps the relatively low frequency of emotional words led their participants to expect emotionally neutral words, making the first presentation of a threat word relatively captivating when it arrived. It is also possible that, due to context effects, threat words may not be as salient when embedded in a list with sexual words. However, it is unlikely that the present overall null effect with threat words is due solely to the presence of sexual words, given that Harris and Pashler also observed no attention capture for threat words overall, but had no sexual words in their experiment. It is worth noting, though, that the context in which specific words are shown (i.e., what other words are presented when, and how often) may play a role in determining which words capture attention and for how long.

It could be argued that the attentional capture for the sexual words is the result of a category effect, in that repeated exposure to sexual words primes attention to that category, thereby increasing the interference across trials. If repeated category priming leads to attentional capture, the school words, which also formed a tight category, should show a similar increase in parity RT as the sexual words. However, the school words did not show any evidence of attentional capture, providing evidence that the presence of a thematic category is not sufficient to capture attention.

Using the school category also allowed us to investigate the hypothesis that personal relevance may explain the 
attentional capture of emotional stimuli. Participants in the study were young adult university students - a group for whom sexual and school words would both likely be concern relevant. However, parity RTs increased for sexual words but not for school words, providing evidence that concern relevance cannot explain the pattern of results.

To investigate the question of why sexual words elicit increases in parity RTs, we examined valence and arousal word ratings. The most arousing words led to the longest RTs, but there was no relationship between participants' valence ratings of the words and parity RTs. Arousal ratings were higher for the sexual words than for the threat words, which may explain why sexually explicit words appear to have more power to capture attention than threat words in this paradigm and others, when using nonselected university participants.

The arousal level of the word appears to influence parity RTs by increasing the likelihood that the word will be encoded into memory. A mediated relationship was observed between arousal ratings and parity RT, whereby higher arousal ratings increase the likelihood of word encoding into memory, and this encoding then disrupts parity performance. Arousal ratings had no effect on parity RTs if the word was not encoded, suggesting that high arousal is necessary but not sufficient to disrupt parity performance. The present results can be explained nicely by the binding hypothesis of Mackay et al. (2004). According to these authors, activation of highly arousing meanings (e.g., to taboo words) facilitates the binding of meaning to other contextual features of the words, such as font, color, or location. Because attention is needed for binding, a facilitation in binding for features of sexual words would also require attentional engagement of the sexual words, thus leaving less attention for the digit task. A facilitation in binding would also provide more retrieval cues for these words, and thus lead to superior memory for these highly arousing words in a subsequent surprise memory task. In Mackay et al.'s model, sexual words are assumed to receive superior binding by triggering emotional reactions via the amygdala, which then triggers the hippocampus to activate attention to bind the arousing stimuli to their context, thus enabling superior encoding and retrieval.

In light of the present results, Harris and Pashler's (2004) claim that emotional stimuli do not capture attention beyond a single trial in normal populations seems premature. Clearly, what does matter is what is meant by "emotional" stimuli. Although in agreement with the findings of Harris and Pashler that negative/threat-related stimuli do not capture attention in normal populations, the present investigation provides evidence that sexually explicit words can in fact capture attention over many trials.

\section{AUTHOR NOTE}

This work was made possible by an operating grant from NSERC and an infrastructure grant from the CFI and OIT to the second author. Correspondence concerning this article should be addressed to K. M. Arnell,
Department of Psychology, Brock University, St. Catharines, ON, L2S 3A1 Canada (e-mail: karnell@brocku.ca).

Note-Accepted by David A. Balota's editorial team.

\section{REFERENCES}

Anderson, A. K. (2005). Affective influences on the attentional dynamics supporting awareness. Journal of Experimental Psychology: General, 134, 258-281.

Arnell, K. M., Killman, K. V., \& FiJavz, D. (in press). Blinded by emotion: Target misses follow attentional capture by arousing distractors in RSVP. Emotion.

DALgLeish, T. (1995). Performance on the emotional Stroop task in groups of anxious, expert, and control subjects: A comparison of computer and card presentation formats. Cognition \& Emotion, 9, 341-362.

DiJKsterhuis, A., \& AarTs, H. (2003). On wildebeests and humans: The preferential detection of negative stimuli. Psychological Science, 14, 14-18.

HaRRIs, C. R., \& PASHLER, H. (2004). Attention and the processing of emotional words and names: Not so special after all. Psychological Science, 15, 171-178.

KuČERA, H., \& FrANCIS, W. N. (1967). Computational analysis of presentday American English. Providence, RI: Brown University Press.

Larsen, R. J., Mercer, K. A., \& Balota, D. A. (2006). Lexical characteristics of words used in emotional Stroop experiments. Emotion, 6, 62-72.

MacKay, D. G., Shafto, M., Taylor, J. K., Marian, D. E., Abrams, L., \& DYER, J. R. (2004). Relations between emotion, memory, and attention: Evidence from taboo Stroop, lexical decision, and immediate memory tasks. Memory \& Cognition, 32, 474-488.

McKenna, F. P., \& Sharma, D. (1995). Intrusive cognitions: An investigation of the emotional Stroop task. Journal of Experimental Psychology, 21, 1595-1607.

Schneider, W., Eschman, A., \& Zuccolotto, A. (2002). E-Prime user's guide [Computer software]. Pittsburgh: Psychology Software Tools, Inc.

Williams, J. M. G., Mathews, A., \& MacLeod, C. (1996). The emotional Stroop task and psychopathology. Psychological Bulletin, 120, 2-24.

WOLFORD, G., \& MORRISON, F. (1980). Processing of unattended visual information. Memory \& Cognition, 8, 521-527.

\section{NOTE}

1. Threat/fear and neutral words were randomly selected from the McKenna and Sharma (1995) set as per Harris and Pashler's (2004) experimental design.

\section{ARCHIVED MATERIALS}

The following materials and links can be accessed through the Psychonomic Society's Norms, Stimuli, and Data Archive, www .psychonomic.org/archive.

To access these files, search the archive for this article using the journal (Psychonomic Bulletin \& Review), the first author's name (Aquino), and the publication year (2007).

FILE: Arnell-PB\&R-2007.zip.

DESCRIPTION: The compressed archive file contains two files:

wordinfo.pdf, containing a PDF file of the word stimuli presented as distractors, their arousal and valence ratings, and the digit-parity RTs on trials in which the words were presented.

wordinfo.txt, containing a text file of the word stimuli presented as distractors, their arousal and valence ratings, and the digit-parity RTs on trials in which the words were presented.

AUTHOR’s E-MAIL ADDRESS: karnell@brocku.ca.

(Manuscript received July 19, 2005; revision accepted for publication July 18, 2006.) 